\title{
Las inteligencias, emociones y cronotipo, ¿̇explican el desempeño académico en universitarios?
}

\section{Do the intelligences, emotions and chronotype, explain academic performance in university students?}

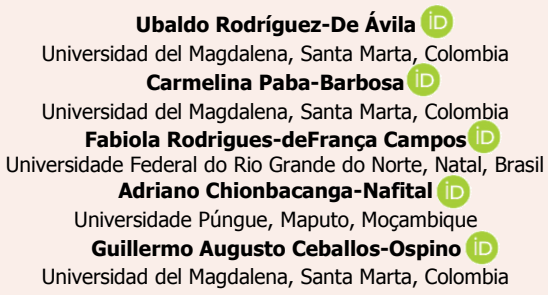

Ubaldo Rodríguez-De Ávila

Universidad del Magdalena, Santa Marta, Colombia Carmelina Paba-Barbosa

Universidad del Magdalena, Santa Marta, Colombia

Fabiola Rodrigues-deFrança Campos i

Universidade Federal do Rio Grande do Norte, Natal, Brasil

Adriano Chionbacanga-Nafital

Universidade Púngue, Maputo, Moçambique

Guillermo Augusto Ceballos-Ospino

Universidad del Magdalena, Santa Marta, Colombia

\section{Resumen}

Objetivo: Identificar cómo las inteligencias, emociones y el cronotipo explican el desempeño académico en universitarios. Método: Estudio de tipo cuantitativo, exploratorio, con una muestra de 493 sujetos universitarios (edad $M=22.06 \pm 4,59$ ); $32 \%$, hombres y $68 \%$, mujeres. Resultados: Se encontró asociación entre el desempeño académico y la edad $[X 2=7.386(2), p=.02]$; el desempeño académico y el afecto positivo $[X 2=6.623(2), p=.03]$; calidad de sueño y síntomas depresivos $(R 2=.211)$, síntomas depresivos e ideación suicida $(R 2=.408)$; cronotipo y síntomas depresivos e ideación suicida $(R 2=.113 ; \mathrm{R} 2=.106$, respectivamente). Mediante regresión multivariada, se identificó que solo el $1,1 \%$ de la varianza del desempeño académico es explicada por la edad $[\mathrm{F}=6.512(1), \mathrm{p}=.01]$. Discusión y Conclusiones: Se concluyó que las variables emocionales, las inteligencias múltiples, la ideación suicida y el cronotipo no explican el desempeño académico en estudiantes universitarios. Se sugiere que el desempeño académico, en tanto dependiente de factores como inteligencias, emoción y cronotipo, no puede ser explicado convenientemente.

Palabras clave: Inteligencias, emoción, cronotipo, desempeño académico.

\begin{abstract}
Objective: Identify if the intelligences, emotions and chronotype explain the Academic Performance in university students. Method: Quantitative, exploratory study, with a sample of 493 university subjects (age $M=22.06 \pm 4.59$ ) $32 \%$ men and $68 \%$ women. Results: Associations were found between Academic Performance and Age $[X 2=7.386(2), p=.02]$; Academic Performance and Positive Affection $[X 2=6.623(2), p=.03]$; Sleep quality and depressive symptoms $(R 2=.211)$, depressive symptoms and suicidal ideation $(R 2=.408)$; Chronotype and depressive symptoms and suicidal ideation $(\mathrm{R} 2=.113 ; \mathrm{R} 2=.106$ respectively). Through a multivariate regression, it was identified that only $1.1 \%$ of the Academic Performance variance is explained by Age $[F=6.512(1), p=.01]$. Discussion y Conclusion: It is concluded that emotional variables, multiple intelligences, suicidal ideation and chronotype do not explain Academic Performance in university students. It is argued that Academic Performance as dependent on factors such as intelligences, emotion and chronotype cannot be explained properly.
\end{abstract}

Keywords: Intelligences, emotion, chronotype, academic performance.
Open Access:

ISSN: $0124-2121$ E-ISSN: $2665-2420$

ARTÍCULO RSULTADO DE

INVESTIGACION

By Educación y Humanismo

Editor:

Patricia Martínez Barrios Universidad Simón Bolívar

Correspondencia:

Ubaldo Rodríguez

rodriguez.ubaldo@gmail.com

Recibido: $19-09-2019$

Aceptado: 23-10-2019

Enlíneadesde: 02-12-2019

DOI:

10.17081/eduhum.22

38.3636

Cómo citar este artículo (APA): Rodríguez-De Ávila, U., Paba-Barbosa, C., Rodrigues-deFrança, F., Chionbacanga-Nafital, A. \& Ceballos-Ospino, G. (2020). Las inteligencias, emociones y cronotipo, ¿explican el desempeño académico en universitarios?. Educación y Humanismo, 22(38), 1-21. DOI: 10.17081/eduhum.22.38.3636 


\section{Introducción}

El Cronotipo ( $\mathrm{Cr}$ ) se define como la temporización interna marcada por el punto medio del sueño. Este varía de persona a persona, debido a la diferencia individual de organización temporal (tarde o temprano) de las diversas actividades diarias, preferencias diurnas 0 fenotipo circadiano (Leocadio-Miguel et al, 2017; Markarian, Warnke \& Pickett, 2017; Rhee, Lee, Rex \& Kripke, 2012; Randler \& Rahafar, 2017; Roenneberg, Allebrandt, Merrow \& Vetter, 2012; Wehrwein \& Joyner, 2013). En la adolescencia, el $\mathrm{Cr}$ se relaciona con cambios en la cognición, la emoción y la fisiología, entre estos, uno de los cambios más importantes es un retraso dramático en el tiempo diario del ciclo de sueño-vigilia, correlacionado con la maduración puberal (Hummer \& Lee, 2016; Reichert et al, 2017; Sheldon, 2014; Touitou, Touitou \& Reinberg, 2017).

Los sujetos con $\mathrm{Cr}$ matutino tienen una ventaja académica en relación con los vespertinos (Enright \& Refinetti, 2017, Van Der Vinne et al, 2015), pues se sabe que el desempeño académico se relaciona con el Cr (Horzum, Önder \& Besoluk, 2014; Beşoluk, Önder \& Deveci, 2011). En general, los resultados sugieren que los vespertinos presentan un peor desempeño académico, tanto en grados de enseñanza primaria como en universitarios (Tonetti, Natale, \& Randler, 2015; Van Der Vinne et al, 2015), y que los matutinos presentan mejores notas que los vespertinos, por la organización social del tiempo de clases generalmente en horas diurnas (Enright y Refinetti, 2017).

Por otro lado, los trastornos del ciclo sueño y vigilia, así como la Calidad de Sueño (CS) son frecuentes en adultos jóvenes universitarios, y su presencia puede condicionar alteraciones diurnas y pobre rendimiento académico. Esta poca CS, tan frecuente en la población universitaria, está asociada al bajo Desempeño Académico (DA) (Lucero et al, 2014). En efecto, algunos estudios han demostrado que la baja CS, determinada por baja eficiencia del mismo y la somnolencia, influyen en un menor DA al final del semestre en estudiantes de medicina (Machado-Duque, Echeverri-Chabur \& Machado-Alba, 2015; Niño et al, 2018; Prichard \& Hartmann, 2019).

Se debe tomar en cuenta que el intelecto humano es un conjunto de dispositivos computacionales semiautónomos, capaces de procesar ciertos tipos de información en cierto tipo de formas. Esto es lo que se define como Inteligencias Múltiples (IM) (Gardner, 2006, 1985), que son importantes en el proceso de aprendizaje y el DA (Akkuzu \& Akçay, 2014; Almeida, Prieto, Ferreira, Bermejo, Ferrando, \& Ferrándiz, 2010; Ayoub \& Aljughaiman, 2016; Branton \& Karanian, 2017; Hernández-Torrano, Ferrándiz, Ferrando, Prieto \& Fernández, 2014; Maguirea, Egana, Hylanda \& Maguireb, 2016; Perera, 2015; Powell, Nettelbeck \& Burns, 2017; Viguer, Cantero \& Bañuls, 2017). En 224 publicaciones, entre 1983 y 2015, se ha propuesto la teoría de la IM en la implementación de currículos, identificando modelos de enseñanza basados en esta teoría, así como evidencias de la proyección práctica basada en IM (Díaz-Posada, Varela-Londoño \& Rodríguez-Burgos, 2017; Oliveira-Jorge, Esgalhado \& Pereira, 2016). 
En cuanto a las emociones, hoy se sabe que estas son experiencias subjetivas que las personas representan con cientos de términos semánticos (Cowen \& Keltner, 2017), como producto de eventos ricos en contenido psicológico, causalmente constituidos por procesos neurobiológicos (Barrett \& Wager, 2006) que impactan la personalidad (Purnamaningsih, 2017; LeDoux, Phelps \& Alberini, 2016). También se conciben como un factor en la organización de la conciencia con influencia en el comportamiento (Izard, 2007), con estados o sentimientos programados de manera innata en las áreas subcorticales del cerebro (LeDoux \& Brown, 2017) y que incluyen dos grandes ejes, emociones positivas y negativas (Desatnik et al, 2017; Meiselman, 2017; Ou \& Verhoef, 2017; Rico, Alzate \& Sabucedo, 2017; Scott-Parker, 2017).

La Teoría de la Selectividad Socioemocional y del Procesamiento Cognitivo Dirigido a Objetivos (Fung \& Carstensen, 2003) propone que los cambios sistemáticos en las metas son una función de los horizontes temporales percibidos, que la juventud tiende a experimentar por la exploración y el aprendizaje (Carstensen \& DeLiema, 2018); por esto, todo proceso emocional está entrelazado con la percepción, la cognición, la motivación, el comportamiento y el DA (Bota, Sporns \& Swanson, 2015; Markov et al, 2013; Oh et al, 2014; Pessoa, 2013, 2018).

Finalmente, la Ideación Suicida (IS), se considera como el primer paso del eslabón que termina en el suicidio consumado, que afecta la vida escolar del sujeto y tiene incidencia en la salud pública (Aalsma \& Brown, 2008; Arroyave, 2012; Hawkert \& Boulton, 2000; Kim \& Levental, 2008; Valadez, Amezcua, González, Montes \& Vargas, 2011; Wahlbeck, 2015). También se ha determinado que de 10 estudiantes entre los 15 y 16 años, ocho presentan IS (González, Gil, Hernández \& Henao, 2015), lo que conlleva a un riesgo de salud mundial (Ceballos et al, 2015; Large et al, 2017; Organización Mundial de la Salud, 2012, 2014, 2015a, 2015b; Stenbacka, Moberg, Romelsjö \& Jokinen, 2012; Ventura-Juncá et al., 2010; Nock et al., 2013). Se ha encontrado que el DA influye sobre la IS (Palacios, Andrade \& Betancourt, 2006), debido primordialmente a que las dificultades académicas predisponen al fenómeno suicida (Palacios \& Andrade, 2007); sin embargo, hay quienes afirman que la IS no se relaciona con el DA (Wu, Wu, Feng, Meng \& Chen, 2012).

Otros estudios han encontrado asociación entre IS y sexo femenino (Anastasiades, Kapoor, Wootten \& Lamis, 2016; Ávila, 2014) IS y Depresión, control de impulsos, desesperanza, auto concepto o autoestima, tensiones psicológicas, ansiedad y estrés (Ceballos et al. 2015; Farabaugh et al. 2012; Sánchez, Muelas \& García, 2013; Wu, Wu, Feng, Meng \& Chen, 2012; Zhang, Liu \& Sun, 2017).

En este último sentido, la Organización Mundial de la Salud (2012) estima que la depresión afecta a 350 millones de personas, considerándose un problema de salud pública, con mayor diferencia significativa en las mujeres que en varones adolescentes (Allison, Roeger, Graham \& Keeves, 2001; Echeverría, 2015); también se asocia con la IS (Flores, Perales \& Gómez, 2016; González, Martínez, García \& Ferrer, 2017; Herrero \& Gracia, 2007; Pardo, Sandoval \& Umbarila, 2004; Rosas, Yampufé, López, Carlos \& Sotil, 2011) y es responsable de una alta proporción de suicidios en individuos entre los 5 y los 80 años, independiente del sexo, 
condición socioeconómica y de la región de la cual provenga (Ferrari et al., 2014).

Algunos estudios comprueban la relación entre la edad de la adolescencia, síntomas depresivos y DA (Reyes-Rodríguez, Rivera-Medina, Cámara-Fuentes, Suárez-Torres \& Bernal, 2013), que pueden conllevar a la IS (Çelik, Ceylan, Ünsal y Çağan, 2018; Fleming, Edwin, Hayes, Locke \& Lockard, 2018; Keyes, Eisenberg, Perry, Dube \& Dhingra, 2012; Kernan, 2019; Villardón, 2009). No obstante, otro estudio en estudiantes universitarios no halló relación entre la IS y síntomas depresivos con el DA, pero sí entre las dos primeras (Nyer et al., 2013). También se ha encontrado relación entre las primeras horas de clase en los adolescentes, la depresión y otras conductas de riesgo para la salud mental y física (Wahlstrom \& Owens, 2017).

En todo caso, se sabe que el objetivo fundamental de todo proceso pedagógico es el DA, y siendo la formación universitaria una actividad importante para el desarrollo integral del ser y la sociedad, el DA se volcó como el punto de confluencia de todas las fuerzas impulsoras de progreso integral. Por esto, el DA funciona como una red de articulaciones cognitivas de cualidad y de cantidad que involucra factores de medición y predicción de la experiencia educativa, cuya comprensión está claramente demarcada en un modelo de interacción de factores endógenos y exógenos al individuo (Fajardo, Maestre, Felipe, León \& Polo, 2017; Ingram et al, 2016; Phillips et al, 2017). Por consiguiente, el presente trabajo se planteó la hipótesis de que el DA, cuantificado en notas, como define el sistema educativo convencional, puede ser afectado por factores cognitivos, emocionales y del cronotipo, pero ello no explica el DA como un todo holístico en el sistema educativo convencional.

\section{Método}

\section{Población y muestra}

La muestra se compuso de 493 estudiantes universitarios, de segundo semestre en adelante, en diferentes facultades de la Universidad del Magdalena (Santa Marta, Colombia). De ellos, el $17 \%$ cursaba entre el segundo y el cuarto semestre; el 44\%, entre quinto y séptimo semestre, y el 39\% cursaba semestres superiores a séptimo. La edad media fue de 22.06 44.59, distribuida así: El 57\% en la faja de edad de hasta 21 años (Adolescentes), el $37 \%$ de entre 21 y 30 años de edad (Adulto joven) y el $6 \%$, mayor de 30 años. Por otra parte, el $32 \%$ son hombres y el $68 \%$, mujeres.

El criterio de inclusión fue el de ser estudiante matriculado en el segundo período de 2018 y que firmaran la participación libre y voluntaria. Se excluyeron así los estudiantes que presentaban alguna capacidad diferencial que les impidiera diligenciar por sí mismo los cuestionarios. Tales cuestionarios fueron enviados y diligenciados vía Web.

El DA fue consultado directamente con la dependencia encargada en la institución universitaria. El DA es acumulativo, y se estipuló a partir del cálculo de la sumatoria de todas las notas de todos los semestres dividido por el número de créditos cursados. 


\section{Instrumentos}

Cuestionario de Matutinidad/Vespertinidad (MEQ) de Horne y Ostberg (1976). Está compuesto por 19 preguntas, cada una de ellas con opciones de respuestas tipo Likert de cinco (5) puntos. Las puntuaciones van de 16 a 86 puntos; las de 41 o menos indican "tipo vespertino". Puntuaciones de 59 o más indica "tipo matutino" y las puntuaciones entre 42 a 58 indican "tipo intermedio". Las puntuaciones permiten clasificar a los individuos en cinco (5) categorías: Vespertino Extremo, Vespertino Moderado, Intermedio, Matutino Moderado y Matutino Extremo.

Como insumo para la presente investigación, se tomaron datos de un estudio piloto realizado con 119 estudiantes universitarios en el periodo 2017-1; el Análisis Factorial confirmó un coeficiente Alfa de $71.4 \%$ ( $a=.714$ ) en un rango que va desde .683 hasta .725 . El Análisis Factorial Confirmatorio reveló un coeficiente $\mathrm{KMO}=.662$ y la prueba de esfericidad de Bartlett presentó $X^{2}=415.078(18) ; p<.05$. El cálculo de las varianzas explicadas fue 20.066. El Chicuadrado de Friedman fue de $X^{2}=518.470(18), p<.05$. Todo lo anterior demuestra que el instrumento tiene un adecuado desempeño psicométrico.

Índice de calidad de sueño de Pittsburgh (IQSP). Es un instrumento de 19 ítem, ampliamente utilizado y validado en diferentes idiomas, incluyendo el español. Se usa para cuantificar la calidad del sueño (Buysse et al., 1989). Los resultados se agrupan en siete componentes. La puntuación total varía de 0 a 21 puntos. Una puntuación de 0 a 4 puntos indica buena calidad de sueño; la puntuación de 5 a 10, mala calidad de sueño; y por encima de 10 puntos se sitúan posibles trastornos del sueño.

EI IQSP ya fue traducido para varios idiomas y es ampliamente utilizado en ambientes de investigación clínica y básica. Por ejemplo, tiene validación para la lengua Curda (Seidi et al, 2019), para el mandarín (Zhu, Chang, Mary \& Kapella, 2019), para el portugués de Brasil (Naimaier et al, 2011; Passos et al, 2017), para el alemán (Hinz et al, 2016). Y, obviamente, también fue validado en la versión castellana en diferentes momentos: En Perú hubo un reporte de validación en 2015 (Luna-Solis, Robles-Arana \& Agüero-Palacios, 2015); un reporte más antiguo, reportó validación del PSQI con una consistencia interna mediada por coefiente Aplha de Cronbach de $\mathrm{a}=.81$; el coeficiente Kappa de .61; una sensibilidad de $88.63 \%$; y Especificidad de $74.99 \%$, con valor predictivo de la prueba positiva de 80,66 (Macías \& Royuela, 1997). Para Colombia, se reportó una consistencia interna de Cronbach de $a=.78$; además se encontró diferencia en las puntuaciones de ancianos comparados con jóvenes ( $U$ de Mann Whitney: $Z=-1.926, p=.0542$ ), pero no se encontró diferencia por sexo (EscobarCórdoba \& Eslava-Schmalbach, 2005).

Escala de Múltiples Inteligencias (IIM). Consta de 48 ítems reunidos para 8 factores, que corresponden a cada tipo de inteligencia (Lingüística, Lógico-Matemática, Visual-Espacial, Kinestésica o Corporal-cinética, Musical, Interpersonal, Intrapersonal y Naturalista). El 
instrumento tiene 5 niveles de respuesta tipo Likert. Cuánto más se acerca a 5, más define al estudiante, y cuánto más se acerca a 1 no define al estudiante. Las puntuaciones para cada inteligencia se configuran de la siguiente forma: Bajo para las puntuaciones entre 1 y 17 ; Medio para puntuaciones entre 18 y 23; Alto para puntuaciones entre 24 y 28; y Superior para puntuaciones igual o mayores a 30.

Se construyó la escala y se hizo un estudio con 1501 estudiantes universitarios en el periodo 2017-1. Sus 48 componentes se sometieron a un análisis de Fiabilidad Alpha de Cronbach, dando como resultado una consistencia interna del $86,4 \%(a=.864)$ y un Alpha para elementos tipificados de $a=.873[F=326.264(47) ; p<.05]$, lo que sugiere que el instrumento tiene una validez de constructo y confiabilidad adecuadas y puede ser utilizado conforme fue diseñado.

En el presente estudio, se verificó un Alpha de Cronbach unifactorial adecuado $(a=.895)$, por lo que se decidió utilizar el IIM de manera general. El comportamiento psicométrico demostró, en efecto, que no existe varianza significativa entre las escalas de las Inteligencias Múltiples. De ésta forma, los valores inferiores del IIM corresponden a un nivel inferior, y los valores altos corresponden a un nivel superior en IIM.

Escala de Afecto Positivo y Negativo (AP-AN). Los autores (Zanon et al, 2013) reportaron una consistencia interna de $83 \%$ para AP y $77 \%$ para AN, con población de jóvenes brasileros. Como la original en portugués, la versión en español está compuesta por 20 ítems: 10 referentes a AP y 10 para AN. Las opciones de respuesta están presentadas en una escala Likert de cinco puntos. Cuánto más próximo de cinco (5), más el ítem describe al participante, y cuanto más próximo de uno (1), menos lo describe.

En un pilotaje realizado con estudiantes universitarios en 2017-1, el Análisis Factorial Exploratorio confirmó el coeficiente de correlación $a=.78$ para AP, y para AN $a=.779$. El Análisis Factorial Confirmatorio para AP arrojó, además, un coeficiente $\mathrm{KMO}=.770$ $[X 2=266,733(19) ; \mathrm{p}<.05]$ y para el AN se verificó un $\mathrm{KMO}=.758[\mathrm{X} 2=280,451(19) ; \mathrm{p}<.05]$. El cálculo de las varianzas explicadas como resultado del Análisis de Componentes Principales para AP fue de 23.01 y para AN, de 12.93. Además, el análisis exploratorio de validez convergente interna para el constructo de AP y AN mostró correlación significativa negativa para Afecto Positivo y Negativo $(-.336 ; \mathrm{p}<.01)$. Todo esto demuestra el valor psicométrico de la escala.

Escala del Centro de Estudios Epidemiológicos de la Depresión-10 (CES-D-10). Consta de 10 ítems tipo Likert con cuatro opciones de respuesta desde Nunca, A veces, Muchas veces hasta Sempre. En estudio realizado con 350 estudiantes de la ciudad de Santa Marta, ésta escala presentó un alfa de .86 y omega .88 .

Escala de Okasha (1981) para Suicidalidad (IS). Consta de cuatro ítems En un estudio realizado en Santa Marta con 350 estudiantes, esta escala obtuvo un coeficiente alfa de Cronbach de .80 y omega .84 . 


\section{Procesamiento estadístico}

El análisis descriptivo incluye observación de frecuencias y porcentajes. Para todas las variables interválicas o proporcionales se estimó la media $(\mathrm{M})$ y desviación estándar $( \pm)$. A estas variables se les aplicó la prueba de Kolmogorov-Smirnov para conocer la distribución de las mismas. En todas las variables se comprobó la distribución no normal y el análisis bivariado incluyó la estimación de correlaciones de Spearman. Para variables dicotómicas, como el sexo, se calculó la correlación de tau de Kendall. En este análisis se considerará el DA como variable dependiente, y el cronotipo (Cr), calidad de sueño (CS), inteligencias múltiples (IIM), afecto positivo y negativo (AP y AN), síntomas depresivos (SD) e Ideación Suicida (IS) como variables independientes.

Para todas las variables se verificó la varianza de las categorías intra-grupo mediante chi cuadrado $\left(X^{2}\right)$, y la varianza inter-grupos, mediante el test de Kruskal-Wallis. El análisis multivariado incluyó un modelo de regresión lineal (múltiple), con los ajustes en la codificación según la naturaleza de la variable. En este modelo se estimó betas $(\beta)$ estandarizados con intervalos de confianza del $95 \%$ y valores de $R, R$ ajustado, $R^{2}$ y $R^{2}$ ajustado. Se esperó contar con un modelo final que alcance por lo menos un valor de $\mathrm{R}^{2}$ del $50 \%$. Todo el análisis estadístico se realizó en el programa IBM-SPSS versión 22.

\section{Consideraciones éticas}

La investigación se hizo en consonancia con la Resolución 8430 que reglamentó la investigación en salud y con las normas precisas del Colegio Colombiano de Psicólogos, Conforme a la ley 1090 de 2006: se contó con los permisos correspondientes, la firma de consentimiento informado y el manejo confidencial de toda la información recogida. El proyecto fue autorizado por el comité de Ética de la respectiva Universidad.

\section{Resultados}

Mediante análisis descriptivo con la respectiva prueba de normalidad, se constató significancia en distribución No-paramétrica para todas las variables, y a partir del análisis de la varianza intra-grupo, se confirmó una diferencia significativa entre las categorías internas de cada variable (Tabla 1 ).

Tabla 1. Descriptivos, Normalidad y Comparación intra-grupo de las variables de estudio

\begin{tabular}{|c|c|c|c|c|c|c|c|}
\hline & $\mathbf{M}$ & \pm & KS & & $\mathbf{N}$ & $\%$ & $\mathrm{X}^{2}(\mathrm{gl})$ \\
\hline & & & & Adolescente & 280 & 57 & \\
\hline \multirow[t]{3}{*}{ Edad } & 22.06 & 4.59 & $.186^{*}$ & Adulto Joven & 183 & 37 & $193.343(2)^{*}$ \\
\hline & & & & Adulto & 30 & 6 & \\
\hline & & & & & $N=493$ & & \\
\hline \multirow[b]{2}{*}{ Sexo } & & & & Hombre & 159 & 32 & \\
\hline & & & & Mujer & 334 & 68 & $6220(1)^{*}$ \\
\hline
\end{tabular}




\begin{tabular}{|c|c|c|c|c|c|c|c|}
\hline & & & & & 493 & & \\
\hline & & & & Bajo & 315 & 64 & \\
\hline DA & 375.41 & 28.98 & $.077 *$ & Medio & 165 & 33 & $277501(2) *$ \\
\hline & & & & Alto & 13 & 3 & \\
\hline & & & & $\mathbf{N}$ & 493 & & \\
\hline & & & & Inferior & 76 & 16 & \\
\hline IIM & 16.43 & 3.65 & $.069 *$ & Medio & 349 & 71 & $316.951(2)^{*}$ \\
\hline & & & & Alto & 65 & 13 & \\
\hline & & & & $\mathbf{N}$ & 490 & & \\
\hline & & & & Vespertino Extremo & 12 & 2 & \\
\hline & & & & Vespertino Moderado & 59 & 12 & \\
\hline MEQ & 52.05 & 9.76 & $.065^{*}$ & Intermediario & 285 & 58 & $532.142(4) *$ \\
\hline & & & & Matutino Moderado & 127 & 26 & \\
\hline & & & & Matutino Extremo & 10 & 2 & \\
\hline & & & & 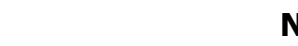 & 493 & & \\
\hline & & & & Facilidad & 144 & 29 & \\
\hline IQSP & 8.71 & 3.68 & $.084 *$ & Dificultad media & 318 & 65 & $254.389(2)^{*}$ \\
\hline & & & & Dificultad severa & 31 & 6 & \\
\hline & & & & & 493 & & \\
\hline CFS-D-10 & 2418 & & 070* & Síntoma medio & 121 & 25 & \\
\hline CES-D-10 & 24.18 & 4.66 & $.0 / 0^{*}$ & Posible Depresión & 372 & 75 & $127.791(1)^{*}$ \\
\hline & & & & $\mathbf{N}$ & 493 & & \\
\hline & & & & Bajo & 23 & 5 & \\
\hline AP & 36.24 & 8.05 & $.094 *$ & Medio & 86 & 17 & $452.523(2)^{*}$ \\
\hline & & & & Alto & 384 & 78 & \\
\hline & & & & & 493 & & \\
\hline & & & & Bajo & 24 & 5 & \\
\hline AN & 34.46 & 7.60 & $.065^{*}$ & Medio & 122 & 25 & $333.789(1)^{*}$ \\
\hline & & & & Alto & 347 & 70 & \\
\hline & & & & & 493 & & \\
\hline & & & & Baja & 111 & 22 & \\
\hline IS & 4.07 & 3.50 & $.129 *$ & Media & 181 & 37 & $27.181(2)^{*}$ \\
\hline & & & & Alta & 201 & 41 & \\
\hline & & & & & 493 & & \\
\hline
\end{tabular}

M: Media. 土: Desviación Estándar. KS: Prueba de normalidad Kolmogorov-Smirnov. X2: Chi Cuadrado. gl: Grados de Libertad. * $\mathrm{p}<.05$. Para todos los casos de KS, gl=490. El nivel de significancia (p) de la prueba KS, sugiere No Normalidad para todos los casos. El nivel de significancia ( $p$ ) para la prueba X2 para muestras no paramétrica, sugiere diferencia significativas intra-grupo, para todos los casos Fuente: Elaboración propia (2019).

Se encontraron, además, 24 correlaciones significativas entre las diferentes variables (Tabla 2).

Tabla 2. Coeficientes de Correlación y Determinación

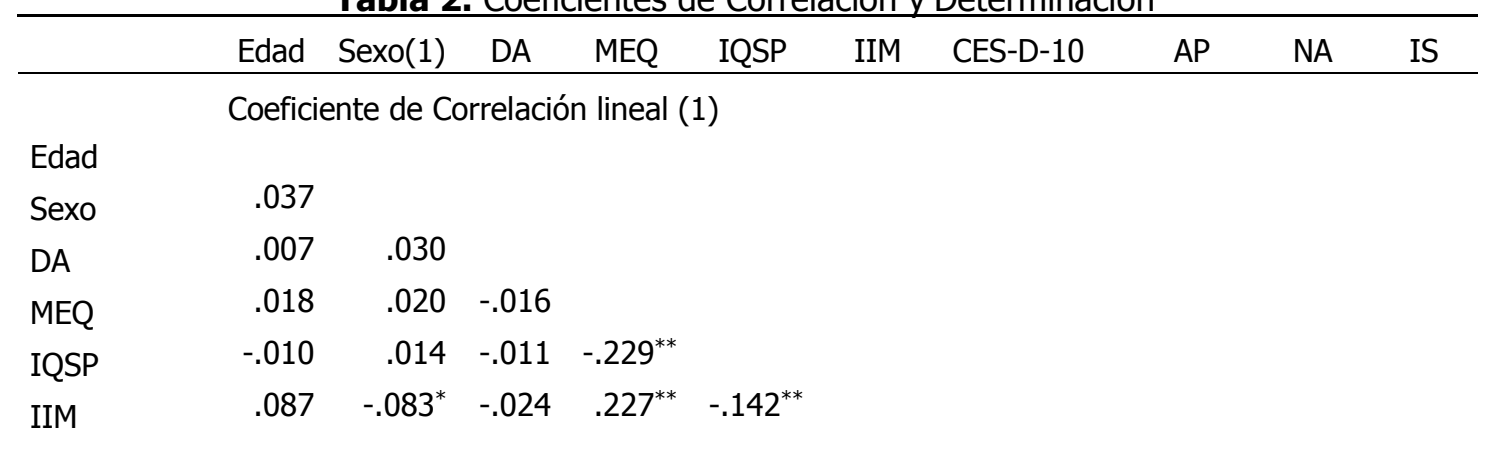




$\begin{array}{llllllllll}\text { CES-D-10 } & .014 & .102^{* *} & .007 & -.326^{* *} & .471^{* *} & -.211^{* *} & & & \\ \text { AP } & .052 & -.028 & -.059 & .298^{* *} & -.411^{* *} & .578^{* *} & -.440^{* *} & & \\ \text { AN } & -.011 & .165^{* *} & .011 & -.214^{* *} & .398^{* *} & -.203^{* *} & .589^{* *} & -.404^{* *} & \\ \text { IS } & .103^{*} & .017 & .036 & -.308^{* *} & .426^{* *} & -.246^{* *} & .635^{* *} & -.475^{* *} & .404^{* *}\end{array}$

Distancia Vectorial Estimada $\left(R^{2}\right)$

\begin{tabular}{|c|c|c|c|c|c|c|c|}
\hline & $\mathrm{R}^{2}$ & & $\mathrm{R}^{2}$ & & $\mathrm{R}^{2}$ & & $\mathrm{R}^{2}$ \\
\hline EDAD - IS & .017 & MEQ - AP & .052 & IQSP - AP & .168 & AP - AN & .171 \\
\hline SEXO - CES & .000 & MEQ - AN & .095 & IQSP - AN & .168 & AP - IIM & .371 \\
\hline SEXO - AN & .000 & MEQ - IIM & .055 & IQSP - IIM & .026 & AP - IS & .233 \\
\hline SEXO IIM & .002 & MEQ - CES & .113 & IQSP - CES & .211 & AN - IIM & .054 \\
\hline IIM - CES & .054 & MEQ - IQSP & . 060 & IQSP - IS & .178 & AN - CES & .345 \\
\hline IIM - IS & .068 & MEQ - IS & .106 & CES - IS & .408 & AN - IS & .154 \\
\hline
\end{tabular}

(1). La correlación realizada para la variable sexo fue la de tau_b de Kendall (tb); para el resto de las variables se usó la Correlación de Spearman (rho). * $\mathrm{p}<.05$. ** $\mathrm{p}<.01$. Se resalta en negrita el coeficiente de determinación con potencial explicativo de la varianza mayor al $10 \%$, entre las variables asociadas.

Fuente: Elaboración propia (2019).

Por otra parte, el análisis de la varianza permitió identificar diferencias significativas en la distribución de DA, Edad y AP. De la misma manera, se identificó diferencia significativa para la variable Sexo, asociada a IIM, SD y AN (Tabla 3 ).

Tabla 3. Análisis de la Varianza para los factores Edad, Sexo y DA

\begin{tabular}{|c|c|c|c|c|c|c|}
\hline \multirow{2}{*}{ Variable } & \multicolumn{2}{|l|}{ Edad } & \multicolumn{2}{|l|}{ Sexo } & \multicolumn{2}{|l|}{ DA } \\
\hline & $\mathrm{X}^{2}(\mathrm{gl})$ & $p$ & $\mathrm{X}^{2}(\mathrm{gl})$ & $p$ & X2(gl) & $p$ \\
\hline Edad & ----- & ---- & ---- & ---- & ---- & ---- \\
\hline Sexo & $1.208(2)$ & .54 & ----- & ----- & ----- & ----- \\
\hline DA & $7.386(2)$ & .02 & $.660(1)$ & .41 & ----- & ---- \\
\hline IIM & $2.245(2)$ & .32 & $4.670(1)$ & .03 & $1.017(2)$ & .6 \\
\hline MEQ & $.077(2)$ & .96 & $.290(1)$ & .59 & $4.983(2)$ & .08 \\
\hline IQSP & $.657(2)$ & .72 & $.143(1)$ & .7 & $.869(2)$ & .64 \\
\hline CES-D-10 & $.404(2)$ & .81 & 7.271(1) & .01 & $3.359(2)$ & .18 \\
\hline AP & $3.699(2)$ & .15 & $.562(1)$ & .45 & $6.623(2)$ & .03 \\
\hline AN & $5.554(2)$ & .06 & $19.275(1)$ & $\begin{array}{r}< \\
.01\end{array}$ & $.353(2)$ & .83 \\
\hline IS & $2.484(2)$ & .28 & $.189(1)$ & .664 & $2.505(2)$ & .28 \\
\hline
\end{tabular}

$\mathbf{X}^{2}$ : Chi cuadrado de Kruskal-Wallis. gl: Grados de Libertad. Resaltado en negrita las diferencias significativas. CES-D-10: Síntoma Depresivo (SD).

Fuente: Elaboración propia (2019).

Se identificaron diferencias significativas en la varianza de las variables Edad y AP. Al respecto, se realizó otra prueba de varianza para estas, con el fin de identificar la independencia de estas variables en sus categorías intra-grupos. Se identificó que las fajas de edad Adolescencia y Adulto Joven están asociadas significativamente al DA $(p<.05)$; sin 
embargo, la relación lineal de Edad solo explica en un 1.3\% el DA mediado por la Distancia Vectorial Estimada $-R^{2}$ (ver figura 1). De la misma forma, el AP-Alto se asocia a DA $(p<.05)$, pero la fuerza de la relación lineal es inversa, solo al 0.7\% (ver Figura 1).

A

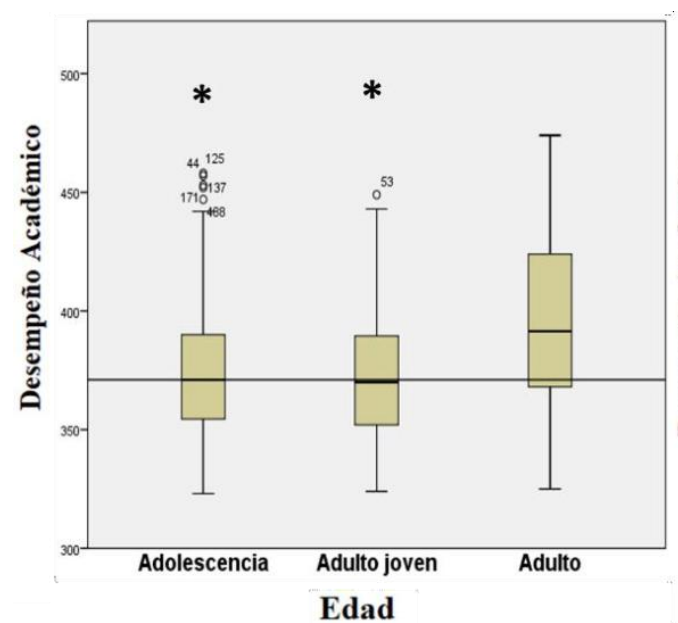

Edad

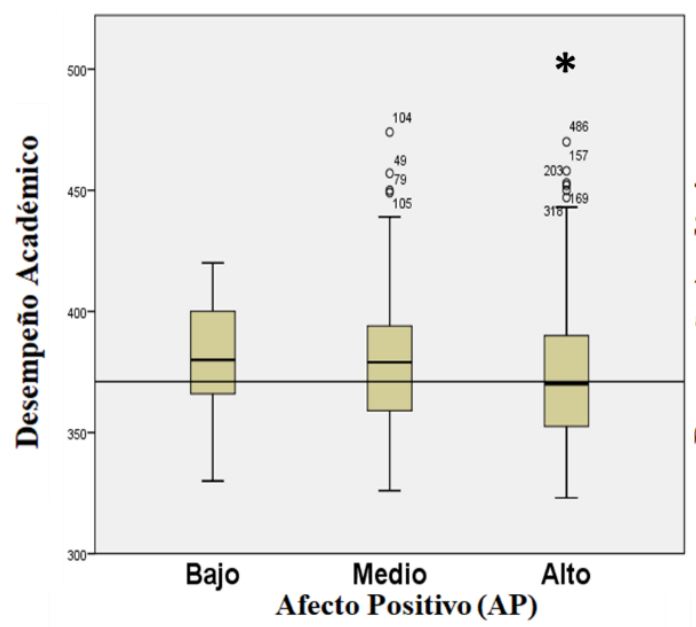

B
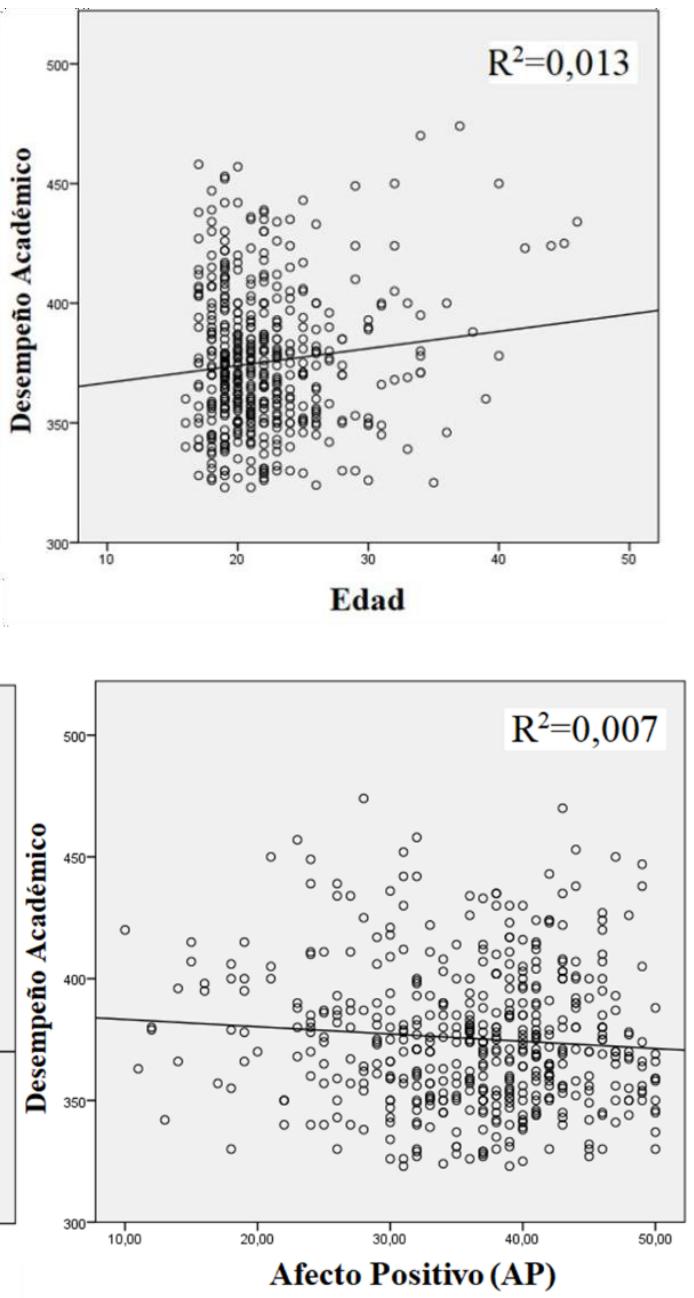

Figura 1. La columna A muestra Blox plot de las variables asociadas con línea de la Mediana de la variable independiente. * muestra significancia en la distribución, lo que sugiere las relación entre las variables DA y AP Alto DA [ $X^{2}=267.979$ (109), $p<$.01]. La columna $B$ muestra la dispersión de Edad y AP respecto del Desempeño Académico (DA), y línea de tendencia con el valor $R^{2}$. La asociación es directa para DA y Edad, e inversa para DA y AP.

Fuente: Elaboración propia (2019).

Igualmente, se identificaron diferencias significativas en las variaciones de IIM, SD y AN asociadas a Sexo. En este sentido, se realizó otra prueba de varianza para estas con el fin de identificar la independencia de las variables asociadas en las categorías intra-grupos de la variable independiente Sexo, identificándose que las categorías de sexo (Hombre y Mujer) están asociadas significativamente a cada una de las variables dependientes $(p<.05)$. Más 
concretamente, la asociación entre la categoría Hombre fue identificada de la siguiente manera: IIM [ $\left.X^{2}=73.266(17), p<.05\right]$, SD $\left[X^{2}=94.289(22), p<.05\right]$, AN $\left[X^{2}=71.648(30), p<\right.$ .05]. De la misma manera, la asociación entre la categoría Mujer fue identificada así: IIM $\left[X^{2}=145.861(18), p<.05\right]$, SD $\left[X^{2}=146.796(22), p<.05\right], A N\left[X^{2}=121.210(34), p<.05\right]$. Sin embargo, no se pudo realizar la prueba de ajuste lineal para determinar la fuerza de la relación, debido a la naturaleza dicotómica de la variable Sexo.

Con el fin de verificar si el DA puede ser explicado y en qué porcentaje, se realizó una regresión lineal múltiple por las diversas variables estudiadas. Mediante el método por pasos, se definió, como criterio de inclusión al modelo, la probabilidad del estadístico $\mathrm{F}$ con valor $\mathrm{p}<=$ .05 y como criterio de exclusión, la probabilidad $(p)$ del estadístico $F$ con valor $p>=.1$. De ésta forma, solo la variable independiente Edad cumplió con el criterio de inclusión. La siguiente es la información de la Regresión Lineal realizada: $\mathrm{R}=.115 ; \mathrm{R}^{2}=.013 ; \mathrm{R}^{2}$ ajustado $=.011$; error típico (ET) de la estimación de $E T=28.890$. Se concluye, entonces, que el $1.1 \%$ de la varianza del DA es explicado por la Edad $[F=6.512(1), p=.01]$. El residual (RS) fue de: [RS=407301.893(488), $\left.\mathrm{M}^{2}=834.635\right]$ y el modelo extraído fue: $Y=359+0,73 X$. Donde $\mathrm{Y}=$ Variable Dependiente (Desempeño Académico); $\beta=$ Parámetro (Constante del DA; 359; 0.73); $\mathrm{X}=$ Variable predictora $\mathrm{o}$ independiente (Edad).

\section{Discusión y Conclusión}

Con los presentes hallazgos, se puede concluir que el DA acumulado no es un reflejo de la inteligencia, de las emociones, de la salud mental o de características fisiológicas. La idea, por tanto, es entender que el DA, tal como las instituciones educativas pretenden entenderlo, es decir, en la nota acumulada a lo largo del tiempo, no es un indicador objetivo para determinar las características individuales, culturales o fisiológicas de los estudiantes. En el DA intervienen múltiples factores internos y externos, que a su vez están afectados por múltiples situaciones que escapan a la observación del profesor y del investigador.

Debe enfatizarse en que el DA ha de ser entendido desde aspectos micro, es decir, contextualizados en la dinámica evolutiva de los estudiantes y del medio que los rodea, así como de los tiempos de la evaluación y de las dinámicas metodológicas de los docentes que intervienen en el DA acumulado de los estudiantes. La mayoría de las investigaciones que encontraron relación entre el DA y las características de $\mathrm{Cr}$, se centraron es periodos cortos de los proceso de enseñanza/aprendizaje, lo cual significa que el DA fue interpretado de manera transversal al estudio realizado. Por eso, investigadores como Hummer y Lee (2016), Sheldon (2014), y Touitou y Reinberg (2017) manifiestan que en la juventud ocurren ajustes y cambios en la cognición, emoción y fisiología. Entre estos, uno de los más importantes es un retraso dramático en el tiempo diario del ciclo de sueño-vigilia, correlacionados con la maduración puberal, sueño y cognición, consolidación de memoria, atención, aprendizaje y salud. Aunque en la presente investigación no encontramos relación alguna entre DA y Cr y 
Calidad de sueño, esto no contradice los resultados de otros estudios, por considerar el desempeño y aprendizaje desde una perspectiva más atómica.

En el presente estudio se constató, eso sí, que los valores inferiores en el MEQ se relacionan con valores altos en el CES-D-10, con valores altos de AN y con valores altos en la escala IS. Lo anterior sugiere que los estudiantes con Cr Vespertino presentan síntomas depresivos altos, mayor prevalencia de afectos negativos y mayor riesgo de IS. Sin embargo, se verificó que el Afecto Positivo puede ser un factor protector para los Cr Vespertinos. Al mismo tiempo, se comprobó una asociación inversa entre las puntuaciones del IQSP y del IIM y de AP; lo que indica que posibles trastornos del sueño afectan el desarrollo de las habilidades de las inteligencias múltiples y son un factor de riesgo para las emociones positivas. En otro sentido, se verificó que las mayores puntuaciones en el IQSP se relacionan positivamente con mayores puntuaciones en el CES-D-10, AN e IS; lo cual indica que, cuando los estudiantes presentan posibles trastornos del sueño o mala calidad de sueño, aparecen riesgos de depresión, de aparición de emociones negativas y de IS.

Los resultados anteriores apoyan las conclusiones de diversos investigadores, cuando expresan que el $\mathrm{Cr}$ es un rasgo individual relacionado también con diferencias individuales como la personalidad, el estado de ánimo, la emoción y la depresión (Markarian, Warnke \& Pickett, 2017; Leocadio-Miguel et al., 2017), sin embargo, en la presente investigación, se aporta un dato interesante referente a la IS, que estudios anteriores no reportan.

Por otro lado, por las correlaciones inversas encontradas, se puede concluir que mayores valores en IMM, son factores protectores para el desarrollo de síntomas depresivos, emociones negativas y, por tanto, de la IS. Al mismo tiempo se encontró una relación directa entre los valores del IIM y AP, por lo que debe interpretarse que el afecto positivo es factor protector y ayuda al desarrollo de las habilidades de las inteligencias múltiples.

De otra parte, el DA no se relacionó con las Inteligencias Múltiples, por lo que no se concuerda con los resultados que manifiestan que el intelecto humano es un conjunto de dispositivos computacionales semiautónomos y evolucionados, para procesar ciertos tipos de información en cierto tipo de formas manifiestos en múltiples inteligencias (Gardner, 2006, 1985), donde cada una de las principales inteligencias está compuesta de subinteligencias. El presente estudio demuestra, por todo ello, que los currículos no pueden basarse en la Inteligencias Múltiples sin modificar la comprensión y medición del DA acumulado, como proponen diversos investigadores (Díaz-Posada et al, 2017; Maguirea et al, 2016; Perera, 2015; Viguer et al, 2017).

Otro hallazgo esperado, alterno de la presente investigación, es la asociación inversa entre los valores de AP y AN, CES-D-10 y AP, y la correlación directa entre CES-D-10 y AN. Esto significa que las emociones afectan los síntomas depresivos en los estudiantes; siendo las emociones positivas un factor protector y el afecto negativo un factor de riesgo para la sintomatología depresiva, lo que concuerda con la idea de que la emoción se entrelaza con 
todos estos dominios mentales a través de la comunicación entre redes (Bota et al, 2015; Markov et al, 2013; Oh et al, 2014; Pessoa, 2018).

Con relación a la asociación entre IS y DA, se ha encontrado que es el DA el que influye sobre la IS y no al revés (Palacios, Andrade \& Betancourt, 2006), es decir, existe asociación entre la IS, intento de suicidio y el DA, debido primordialmente a que las dificultades académicas son un factor predisponente para el fenómeno suicida (Palacios \& Andrade, 2007). En otras palabras, la IS debe ser en este caso una variable dependiente del DA, sin embargo, en el presente estudio no se encontró relación alguna entre estas variables.

El presente estudio apoya, asimismo, las conclusiones de diversos investigadores (Anastasiades et al, 2016; Ávila, 2014), quienes encontraron que la IS se asocia con mayor frecuencia con el sexo femenino, sin embargo, el potencial explicativo de la varianza no permite concluir de manera contundente al respecto.

Al mismo tiempo, este trabajo corrobora los hallazgos de Ceballos et al (2015), quienes encontraron que la IS está relacionada con malas relaciones personales y síntomas depresivos; igualmente, se relaciona con factores emocionales (Zhang et al, 2017) y apoya un estudio según el cual ideación suicida no se relaciona con el DA (Wu et al, 2012).

La investigación concuerda con los resultados de varias otras que encontraron asociación entre la IS y los Síntomas Depresivos (Flores et al, 2016; González et al, 2017; Herrero \& Gracia, 2007; Pardo et al, 2004; Rosas et al, 2011), mas no con investigaciones según las que las dificultades académicas pueden conllevar a la IS (Keyes et al, 2012; Villardón, 2009) o que los síntomas depresivos afectan el DA en los universitarios (Çelik et al, 2018). Y por otro lado, la presente investigación concuerda con Nyer et al (2013), al considerar que en estudiantes universitarios no existe relación entre la IS, síntomas depresivos y el DA, pero sí entre las dos primeras.

Se concluye, en fin, que las variables emocionales, las inteligencias múltiples, la ideación suicida y el cronotipo no explican el desempeño académico en estudiantes universitarios, pues este, por sí solo, no es una medida objetiva para observar inteligencias o emociones. Para futuras investigaciones, se recomienda tener en cuenta la preferencia vocacional como variable moduladora o independiente, así como otras variables sociológicas, psicológicas y económicas del sujeto para identificar asociaciones fuertes con el desempeño académico.

\section{Referencias}

Aalsma, M \& Brown, J. (2008). What is bullying? Journal of Adolescent Health, 2(43), 101-102. Doi: https://doi.org/10.1016/j.jadohealth.2008.06.001

Akkuzu, N. \& Akçay, A. (2014). The design of a learning environment based on the theory of multiple intelligence and the study its effectiveness on the achievements, attitudes and 
retention of students. Procedia Computer Science, 3(2011), 1003-1008. Doi: http://doi:10.1016/j.procs.2010.12.165

Allison, S., Roeger, L., Graham, M. \& Keeves, J. (2001). Gender differences in the relationship between depression and suicidal ideation in young adolescents. Aust N Z J Psychiatry, 35(4), 498-503. Doi: https://doi.org/10.1046/j.1440-1614.2001.00927.x

Almeida, L., Prieto, M., Ferreira, A., Bermejo, M., Ferrando, M. \& Ferrándiz, C. (2010). Intelligence assessment: Gardner multiple intelligence theory as an alternative. Learning and Individual Differences, 20(2010), 225-230. Doi: http://doi: 10.1016/j.lindif.2009.12.010

Anastasiades, M.H., Kapoor, S., Wootten, J., \& Lamis, D.A. (2016). Perceived stress, depressive symptoms, and suicidal ideation in undergraduate women with varying levels of mindfulness. Arch Womens Ment Health, 20(1):129-138. Doi: 10.1007/s00737-0160686-5

Arroyave, P. (2012). Factores de vulnerabilidad y riesgo asociados al bullying. Revista CES Psicología, 5(1), 118-125. Recuperado de http://www.redalyc.org/articulo.oa?id=423539529012

Ávila, D. (2014). Nivel de prevalencia de ideas e intentos suicidas en los estudiantes de sexto semestre de seis preparatorias de Montemorelos (Doctoral dissertation). Recuperado de

http://dspace.biblioteca.um.edu.mx/xmlui/bitstream/handle/20.500.11972/641/Tesis\% 20Diana\%20Selene\%20\%C3\%81vila\%20Hern\%C3\%A1 ndez.pdf?sequence=1\&isAllo wed $=\mathrm{y}$

Ayoub, A. \& Aljughaiman, A. (2016). A predictive structural model for gifted students' performance: A study based on intelligence and its implicit theories. Learning and Individual Differences, 51(2016), Doi: http://dx.doi.org/10.1016/j.lindif.2016.08.018

Barrett, L.F. \& Wager, T. (2006). The structure of emotion: evidence from the neuroimaging of emotion. Curr. Dir. Psychol. Sci, (15), 79-85. Recuperado de http://journals.sagepub.com/doi/abs/10.1111/j.0963-7214.2006.00411.x

Beşoluk, Ş., Önder, I., \& Deveci, I. (2011). Morningness-eveningness preferences and academic achievement of university students. Chronobiology International, 28(2), 118125. Doi: https://doi.org/10.3109/07420528.2010.540729

Bota, M., Sporns, O. \& Swanson, L.W. (2015). Architecture of the cerebral cortical association connectome underlying cognition. Proc Natl Acad Sci, 112(16), E2093-E2101. Doi: http://doi:10.1073/pnas.1504394112

Branton, C. \& Karanian, J. (2017). The Neuroscience of Intelligence: Empirical Support for the Theory of Multiple Intelligences?. Trends in Neuroscience and Education, (6), 211-223 Doi: http://dx.doi.org/10.1016/j.tine.2017.02.002

Buysse, D., Reynolds, C., Monk, T., Berman, S. \& Kupfer, D. (1989). The Pittsburgh sleep quality index: A new instrument for psychiatric practice and research. Psychiatry Research, 28(2), 193-213. Doi: https://doi.org/10.1016/0165-1781

Carstensen, L., \& Deliema, M. (2018). The positivity effect: a negativity bias in youth fades with age. Current Opinion in Behavioral Sciences, (19), 7-12. Doi: 
http://dx.doi.org/10.1016/j. cobeha.2017.07.009

Ceballos-Ospino, G., Suarez-Colorado, Y., Suescún-Arregocés, J., Gamarra-Vega, L., González, K. \& Sotelo-Manjarres, P. (2015). Ideación Suicida, Depresión y Autoestima en adolescentes escolares de Santa Marta. Duazary, 12(1), 15-22. Doi: http://dx.doi.org/10.21676/2389783X.1394

Çelik, N., Ceylan, B., Ünsal, A., \& Çağan, Ö. (2018). Depression in health college students: relationship factors and sleep quality. Psychol Health Med, 21, 1-6. Doi: $10.1080 / 13548506.2018 .1546881$

Cowen, A. \& Keltner, D. (2017). Self-report captures 27 distinct categories of emotion bridged by continuous gradients. PNAS, 114(38), E7900-E7909. http://doi:10.1073/pnas.1702247114

Desatnik, A., Bel-Bahar, T., Nolte, T., Crowley, M., Fonagy, P. \& Fearon. P. (2017). Emotion Regulation in Adolescents: An ERP study. Biological Psychology, S0301-0511(17), 30144-30148. http://dx.doi.org/doi:10.1016/j.biopsycho.2017.08.001

Díaz-Posada, L., Varela-Londoño, S.P. \& Rodríguez-Burgos, L.P. (2017). Multiple Intelligences and Curriculum Implementation: Progress, Trends and Opportunities. Revista de Psicodidáctica, 22(1), 69-83. Doi: http://dx.doi.org/doi:10.1387/RevPsicodidact.15614

Echeverría, M. (2015). Prevención de la depresión en jóvenes: enfoque positivo. Recuperado de

https://academicae.unavarra.es/bitstream/handle/2454/18553/Maite\%20Echeverria\%20Aleman.pdf?se quence=1\&isAllowed $=\mathrm{y}$

Enright, T., \& Refinetti, R. (2017). Chronotype, class times, and academic achievement of university students. Chronobiology International, 34(4), 445-450. https://doi.org/10.1080/07420528.2017.1281287

Escobar-Córdoba, F. \& Eslava-Schmalbach, J. (2005). Validación colombiana del índice de calidad de sueño de Pittsburgh. REV NEUROL, 40(3): 150-155. Doi: https://doi.org/10.33588/rn.4003.2004320

Fajardo, F., Maestre, M., Felipe, E., León, B. \& Polo, M. (2017). Análisis del rendimiento académico de los alumnos de educación secundaria obligatoria según las variables familiares. Educación XXI, 20(1), 209-232. Doi: https://doi.org/10.5944/educXX1.14475

Farabaugh, A., Bitran, S., Nyer, M., Holt, D.J., Pedrelli, P., Shyu, I., Hollon, S.D., Zisook, S., Baer, L., Busse, W., Petersen, T.J., Pender, M., Tucker, D.D., \& Fava, M. (2012). Depression and suicidal ideation in college students. Psychopathology, 45(4), 228-234. Doi: 10.1159/000331598

Ferrari, A. J., Norman, R. E., Freedman, G., Baxter, A. J., Pirkis, J. E., Harris, M. G. \& Whiteford, H. A. (2014). The Burden Attributable to Mental and Substance Use Disorders as Risk Factors for Suicide: Findings from the Global Burden of Disease Study 2010. PLoS One, 9(4), e91936. Doi:10.1371/journal.pone.0091936

Fleming, A.R., Edwin, M., Hayes, J.A., Locke, B.D., \& Lockard, A.J. (2018). Treatment-seeking college students with disabilities: Presenting concerns, protective factors, and academic distress. Rehabil Psychol, 63(1):55-67. Doi: 10.1037/rep0000193

Flores, N., Perales, Y. \& Gómez, W. (2016). Depresión e ideación suicida en los adolescentes 
de una Institución Educativa Pública del distrito de Chongos Bajo - Perú 2014. Ágora Rev Cient, 03(01), 265-274. Doi:10.21679/arc.v3i1.50

Fung, H. \& Carstensen, L.L. (2003). Sending memorable messages to the old: age differences in preferences and memory for advertisements. J Pers Soc Psychol, 85(1), 163-178 http://dx.doi.org/10.1037/0022-3514.85.1.163

Gardner, H. (1985). On discerning new ideas in psychology. New Ideas in Psychology, 3(1), 101-104. https://doi.org/10.1016/0732-118X(85)90057-1

Gardner, H. (2006). On failing to grasp the core of MI theory: A response to Visser et al. Intelligence, 34(2006), 503-505. http://doi:10.1016/j.intell.2006.04.002

González-Portillo J, Gil-Arévalo J, Hernández- Botero D. \& Henao-Sánchez LM. (2015). Evaluación de las expectativas negativas y tipo de riesgo suicida en estudiantes de $9^{\circ}$, $10^{\circ}$ y $11^{\circ}$ de una institución educativa del departamento del Quindío. Duazary, 13(1), 7-14. Doi: 10.21676/2389783X.1582

González, M., Martínez, L. \& García \& Ferrer, D. (2017). Funcionamiento familiar e intento suicida en escolares. Revista Cubana de Medicina General Integral, 36(3), 1-17. Recuperado de http://scielo.sld.cu/scielo.php?script=sci_arttext\&pid=S086421252017000300002

Hawker, D. \& Boulton, M. (2000). Twenty years' research on peer victimization and psychosocial maladjustment: A meta-analytic review of cross-sectional studies. The Journal of Child Psychology and Psychiatry and Allied Disciplines, 41(4), 441-455. Doi https://doi.org/10.1111/1469-7610.00629

Hernández-Torrano, D., Ferrándiz, C. Ferrando, M., Prieto, I. \& Fernández, M. (2014). The theory of multiple intelligences in the identification of high-ability students. Anales de psicología, 30(1), 192-200. Doi: http://dx.doi.org/10.6018/analesps.30.1.148271

Herrero, J. \& Gracia, E. (2007). Una medida breve de la sintomatología depresiva (CESD 7). Salud Mental, 30(5) ,40-46. Recuperado de https://www.medigraphic.com/cgibin/new/resumen.cgi?IDARTICULO=16229

Hinz, A., Glaesmer, H., Brähler, El., Löffler, M., Engel, C., Enzenbach, C., Hegerl, U. \& Sander, C. (2016). Sleep quality in the general population: psychometric properties of the pittsburgh sleep quality index, derived from a german community sample of 9284 people. Sleep Medicine, 30(2017), 57-63. Doi: https://doi.org/10.1016/j.sleep.2016.03.008

Horne, J. \& Ostberg, O. (1976). A self-assessment questionnaire to determine morningnesseveningness in human circadian rhythms. International Journal of Chronobiology, (4), 97-100.

Recuperado

de https://www.researchgate.net/publication/22126774_A_Self_Assessment_Questionnai re_to_Determine_Morningness_Eveningness_in_Human_Circadian_Rhythms

Horzum, M. B., Önder, I. \& Beşoluk, Ş. (2014). Chronotype and academic achievement among online learning students. Learning and Individual Differences, (30), 106-111. https://doi.org/10.1016/j.lindif.2013.10.017

Hummer, D.L. \& Lee, T.M. (2016). Daily timing of the adolescent sleep phase: Insights from a cross-species comparison. Neurosci. Biobehav. Rev, 70(2016), 171-181. Doi: http://dx.doi.org/10.1016/j.neubiorev.2016.07.023 
Ingram, K. Ay, A., Kwon, S., Woods, K., Escobar, S., Gordon. \& Jain, K. (2016). Molecular insights into chronotype and time-of-day effects on decision-making. Scientific Reports, 6(29392). Doi: http://dx.doi.org/10.1038/srep29392

Izard, E. (2007) Basic emotions, natural kinds, emotion schemas, and a new paradigm. Perspect Psychol Sci, 2(3), 260-280. http://doi:10.1111/j.1745-6916.2007.00044.x

Kernan, W. (2019). Health-related impediments to learning among dental and oral surgery students. $J$ Prev Interv Community, 47(1):32-44. Doi:10.1080/10852352.2018.1547307

Keyes, C., Eisenberg, D., Perry, G., Dube, S., Kroenke, K, \& Dhingra, S. (2012). The relationship of level of positive mental health with current mental disorders in predicting suicidal behavior and academic impairment in college students. J Am Coll Health, 60(2), 126-33. Doi: $10.1080 / 07448481.2011 .608393$

Kim, Y., \& Leventhal, B. (2008). Bullying and suicide. A review. International Journal of Adolescent Medicine and Health, 20(2), 13-17. Doi: https://doi.org/10.1515/IJAMH.2008.20.2.133

Large, M., Galletly, C., Myles, N., Ryan, C. \& Myles, H. (2017). Known unknowns and unknown unknowns in suicide risk assessment: evidence from meta-analyses of aleatory and epistemic uncertainty. BJPsych Bulletin, (41), 160-163. Doi: http://doi: 10.1192/pb.bp.116.054940

LeDoux, J. \& Brown, R. (2017) A higher-order theory of emotional consciousness. Proc Nat/ Acad Sci USA, (114), E2016-E2025. Recuperado de http://www.pnas.org/content/114/10/E2016.full.pdf

LeDoux, J., Phelps, L., Alberini, C. (2016). What we talk about when we talk about emotions. Cell, (167), 1443-1445. Doi: http://dx.doi.org/10.1016/j.cell.2016.11.029

Leocadio-Miguel, M., Mazzili, F., Lourenção, L. Peixoto, R., Alam, M. Ventura, M. \& Pedrazzoli, M. (2017). Latitudinal cline of chronotype. Scientific Reports, 7(5437), Doi: https://doi:10.1038/s41598-017-05797-w

Lucero, C., Buonanotte, C., Perrote, F., Concari, I., Quevedo, P., Passaglia, G. \& Mauch, P., (2014). Trastornos del sueño-vigilia en alumnos de $5 .^{\circ}$ año de Medicina de la Universidad Nacional de Córdoba y su impacto sobre el rendimiento académico. Neurología Argentina, 6(4), 184-192. Doi: https://doi.org/10.1016/j.neuarg.2014.05.006

Luna-Solis, I., Robles-Arana, Y. \& Agüero-Palacios, Y. (2015). Validación del índice de calidad de sueño de pittsburgh en una muestra peruana. Anales de Salud Mental, 31(2), 2330.

http://www.insm.gob.pe/ojsinsm/index.php/Revista1/article/view/15/14

Machado-Duque, M., Echeverri-Chabur, J. \& Machado-Alba, J. (2015). Somnolencia diurna excesiva, mala calidad del sueño y bajo rendimiento académico en estudiantes de Medicina. Revista Colombiana de Psiquiatría, 44(3), 137-142. Doi: https://doi.org/10.1016/j.rcp.2015.04.002

Macías, J. \& Royuela, A. (1997). La versión española del índice de calidad de sueño de Pittsburgh. Informaciones Psiquiátricas, (146), 465-472. https://www.researchgate.net/publication/258513992_La_version_espanola_del_Indi 
ce_de_Calidad_de_Sueno_de_Pittsburgh

Maguirea, R., Egana, A., Hylanda, P. \& Maguireb, P. (2016). Engaging students emotionally: the role of emotional intelligence in predicting cognitive and affective engagement in higher education. Higher Education Research \& Development, 8(15), 1-15. Doi: http://dx.doi.org/10.1080/07294360.2016.1185396

Markarian, S., Warnke, A.S. \& Pickett, S.M. (2017). Morningness-eveningness and social anxiety: the indirect effect through punishment sensitivity and experiential avoidance. Sleep, 40(1), A418. https://doi.org/10.1093/sleepj/zsx050.1120

Markov, N., Ercsey-Ravasz, M., Van-Essen, D.C., Knoblauch, K., Toroczkai, Z., Kennedy, H. (2013). Cortical high-density counterstream architectures. Science, 342(6158), 1238406. Doi: http://doi:10.1126/science.1238406

Meiselman, H. (2017). Emotion measurement: Theoretically pure or practical?. Food Quality and Preference, (62), 374-375. Doi: http://dx.doi.org/10.1016/j.foodqual.2017.05.011

Naimaier, A., Chaves, S., Santos, L., Giacomolli, E., Da Silva, I., Ferreira, M. \& Menna-Barreto, S. (2011). Validation of the Brazilian Portuguese version of the Pittsburgh Sleep Quality Index. Sleep Medicine (12), 70-75. Doi:http://dx.doi:10.1016/j.sleep.2010.04.020

Niño, J., Barragán, M., Ortiz, J., Ochoa, M. \& González., H. (2018). Factores asociados con somnolencia diurna excesiva en estudiantes de Medicina de una institución de educación superior de Bucaramanga. Revista Colombiana de Psiquiatría, (260), 1-10. Doi: https://doi.org/10.1016/j.rcp.2017.12.002

Nock, M. K., Green, J. G., Hwang, I., McLaughlin, K. A., Sampson, N. A. \& Zaslavsky, A. M. (2013). Prevalence, correlates, and treatment of lifetime suicidal behavior among adolescents: results from the National Comorbidity Survey Replication Adolescent Supplement. JAMA Psychiatry, (70), 300-310. Doi:10.1001/2013.jamapsychiatry.55

Nyer, M., Holt, D.J., Pedrelli, P., Fava, M., Ameral, V., Cassiello, C.F., Nock, M.K., Ross, M., Hutchinson, D. \& Farabaugh, A. (2013). Factors that distinguish college students with depressive symptoms with and without suicidal thoughts. Ann Clin Psychiatry, 25(1):41-49. Recuperado de https://www.ncbi.nlm.nih.gov/pmc/articles/PMC3791316/

Oh, S.W., Harris, J.A., Ng, L., Winslow, B., Cain, N., Mihalas, S., Henry, A.M (2014). A mesoscale connectome of the mouse brain. Nature, 508(7495), 207-214. Doi: http://doi:10.1038/nature13186

Okasha, A., Lotaif, F. \& Sadek, A. (1981). Prevalence of suicidal feelings in a sample of nonconsulting medical students. Acta Psychiatr Scand, (63), 409-415. Recuperado de https://www.ncbi.nlm.nih.gov/pubmed/7315487

Oliveira-Jorge, D., Esgalhado, G. \& Pereira, H. (2016). Inteligência espiritual: Validação preliminar da versão portuguesa da Escala de Inteligência Espiritual Integrada (EIEI). Análise Psicológica, 3(XXXIV), 325-337. Doi: http://doi:10.14417/ap.982

Organización Mundial de la Salud. (2015a). Suicidio. Nota descriptiva No 398. Ginebra: OMS.

Organización Mundial de la Salud. (2015b). Definición del Trastorno Depresivo. Recuperado de http://www.who.int/topics/depression/es/

Organización Mundial de la Salud. (2014). Health for the World's Adolescents. A Second Chance in the Second Decade. Recuperado dehttp://apps.who.int/adolescent/second- 
decade depression_wfmh_2012.pdf?ua=1

Organización Mundial de la Salud. (2012). Depression a Global Public Health Concern. Recuperado http://www.who.int/mental_health/management/depression/who_paper_

Ou, Y. \& Verhoef, P. (2017). The impact of positive and negative emotions on loyalty intentions and their interactions with customer equity drivers. Journal of Business Research 80(2017), 106-115. Doi: http://dx.doi.org/10.1016/j.jbusres.2017.07.011

Palacios, J. \& Andrade, P. (2007). Desempeño académico y conductas de riesgo en adolescentes. Revista de Educación y Desarrollo, (7), 5-16. Recuperado de http://www.cucs.udg.mx/revistas/edu_desarrollo/anteriores/7/007_Palacios.pdf

Palacios, D. J., Andrade, P. P. \& Betancourt, O. D. (2006). "Intento de suicidio y consumo de alcohol en adolescentes". En: Sánchez, A. R., Díaz-Loving, R. y Rivera, A. S. (Eds.). La Psicología Social en México (pp. 174- 180). México: AMEPSO

Pardo, G., Sandoval, A. \& Umbarila, D. (2004). Adolescencia y depresión. Rev Colomb Psicol, (13), 13-28. https://revistas.unal.edu.co/index.php/psicologia/article/view/1204/1755

Passos, MH. Silva, HÁ. Pitangui, AC., Oliveira, VM. Lima, AS. \& Araújo, RC. (2017). Reliability and validity of the Brazilian version of the Pittsburgh Sleep Quality Index in adolescents. J Pediatr (Rio J), 93(2), 200-206. Doi: http://dx.doi.org/10.1016/j.jped.2016.06.006

Perera, H. (2015). The Role of Trait Emotional Intelligence in AcademicPerformance: Theoretical Overview and Empirical Update. The Journal of Psychology, 0(0), 1-23. Doi: http://dx.doi.org/10.1080/00223980.2015.1079161

Pessoa, L. (2018). Understanding emotion with brain networks. Current Opinion in Behavioral Sciences, (19), 19-25. Doi: http://dx.doi.org/10.1016/j.cobeha.2017.09.005

Pessoa, L. (2013). The Cognitive-Emotional Brain: From Interactions to Integration. Cambridge: MIT Press.

Phillips, A., Clerx, W., O`Brien, C., Sano, A., Barger, L., Picard, R. \& Czeisler, C. (2017). Irregular sleep/wake patterns are associated with poorer academic performance and delayed circadian and sleep/wake timing. Scientific Reports, 7(3216). Doi: https://doi.org/10.1038/s41598-017-03171-4

Powell, C., Nettelbeck, T. \& Burns, N. (2017). The incremental validity of intellectual curiosity and confidence for predicting academic performance in advanced tertiary students. Personality and Individual Differences, 116(2017), 51-56. Doi: http://dx.doi.org/10.1016/j.paid.2017.04.011

Prichard, J., Hartmann, M. (2019). Follow-up to Hartmann \& Prichard: Should universities invest in promoting healthy sleep? A question of academic and economic significance. Sleep Health, 5(4), 320-325. Doi: https://doi.org/10.1016/j.sleh.2019.01.006

Purnamaningsih, E. (2017). Personality and Emotion Regulation Strategies .Int. J. of Pysch. Res, 10(1), 53-60. http://doi:10.21500/20112084.2040

Randler, C. \& Rahafar, A. (2017) Latitude affects Morningness-Eveningness: evidence for the environment hypothesis based on a systematic review. Scientific Reports, 7 (39976). On line. http://dx.doi.org/10.1038/srep39976

Reichert, C., Maire, M., Gabel, V., Viola, A., Götz, T., Scheffler, K. \& Schmidt, C. (2017). 
Cognitive brain responses during circadian wake-promotion: evidence for sleeppressure-dependent hypothalamic activations. Scientific Reports, 7(5620). https://doi.org/10.1038/s41598-017-05695-1

Reyes-Rodríguez ML1, Rivera-Medina CL, Cámara-Fuentes L, Suárez-Torres A, \& Bernal G. (2013). Depression symptoms and stressful life events among college students in Puerto Rico. J Affect Disord, 145(3), 324-30. Doi:10.1016/j.jad.2012.08.010

Rhee, M. K., Lee, H. J., Rex, K. M., \& Kripke, D. F. (2012). Evaluation of two circadian rhythm questionnaires for screening for the delayed sleep phase disorder. Psychiatry Investigation, 9(3), 236-244. Doi: https://doi.org/10.4306/pi.2012.9.3.236

Rico, D., Alzate, M. \& Sabucedo, J. (2017). El papel de la identidad, la eficacia y las emociones positivas en las acciones colectivas de resistencia pacífica en contextos violentos. Revista Latinoamericana de Psicología, 49(1), 28-35. Doi: http://dx.doi.org/10.1016/j.rlp.2015.09.013

Roenneberg, T., Allebrandt, K. V., Merrow, M., \& Vetter, C. (2012). Social jetlag and obesity. Current Biology, 22(10), 939-943. Doi: https://doi.org/10.1016/j.cub.2012.03.038

Rosas, M., Yampufé, M., López, M., Carlos, G. \& Sotil, A. (2011). Niveles de depresión en estudiantes de Tecnología Médica. Revista An Fac med, 72(3), 183-186. Recuperado de http://www.redalyc.org/pdf/379/37922112005.pdf

Sánchez, D., Muelas. J., \& García, A. (2013). Variables psicológicas asociadas a la ideación suicida en estudiantes. International journal of psichology ang psichological therapy, 14(2), 278-290. Recuperado de https://www.ijpsy.com/volumen14/num2/388/variables-psicolgicas-asociadas-a-laideaci-ES.pdf

Scott-Parker, B. (2017). Emotions, behaviour, and the adolescent driver: A literature review. Transportation Research Part F Traffic Psychology and Behaviour, 50(2017), 1-37. http://dx.doi.org/10.1016/j.trf.2017.06.019

Seidi, P., Mohammadi, H., Khazaie, H., Abas, N. \& Jaff, D. (2019). Psychometric Properties of the Kurdish Version of Pittsburgh Sleep Quality Index. Sleep Medicine, (63), 75-81. https://doi.org/10.1016/j.sleep.2019.04.022

Sheldon, S. (2014). The Function, Phylogeny and Ontogeny of Sleep. En Sheldon, S., Ferber, R., Kryger, M. \& Gozal, D. Principles and Practice of Pediatric Sleep Medicine. 2da. Ed (p.p. 3-11). London: Elsevier.

Stenbacka, M., Moberg, T., Romelsjö, A. \& Jokinen, J. (2012). Mortality and causes of death among violent offenders and victims-a Swedish population based longitudinal study. J. BMC Public Health, (17), 12-38. https://doi.org/10.1186/1471-2458-12-38

Tonetti, L., Natale, V., \& Randler, C. (2015). Association between circadian preference and academic achievement: A systematic review and meta-analysis. Chronobiology International, 32(6), 792-801. https://doi.org/10.3109/07420528.2015.1049271

Touitou, Y, Touitou, D. \& Reinberg, A. (2017). Disruption of adolescents' circadian clock: The vicious circle of media use, exposure to light at night, sleep loss and risk behaviors. Journal of Physiology-Paris, 110(4), 467-479. Doi: http://dx.doi.org/10.1016/j.jphysparis.2017.05.001

Valadez, I., Amezcua, R., González, N., Montes, R., \& Vargas, V. (2011). Maltrato entre iguales 
e intento suicida en sujetos adolescentes escolarizados. Revista Latinoamericana de Ciencias Sociales, Niñez y Juventud, 2(9), 783-796. Recuperado de http://www.scielo.org.co/pdf/rlcs/v9n2/v9n2a20.pdf

Van Der Vinne, V., Zerbini, G., Siersema, A., Pieper, A., Merrow, M., Hut, R. A. \& Kantermann, T. (2015). Timing of examinations affects school performance differently in early and late chronotypes. Journal of Biological Rhythms, 30(1), 53-60. https://doi.org/10.1177/0748730414564786

Ventura-Juncá, R., Carvajal, C., Undurraga, S., Vicuña, P., Egaña, J. \& Garib, M.J. (2010). Prevalencia de ideación e intento suicida en adolescentes de la Región Metropolitana de Santiago de Chile. Revista médica de Chile, (138), 309-315. http://dx.doi.org/10.4067/S0034-98872010000300008

Viguer, P., Cantero, M. \& Bañuls, R. (2017). Enhancing emotional intelligence at school: Evaluation of the effectiveness of a two-year intervention program in Spanish preadolescents. Personality and Individual Differences, 113(2017), 193-200. http://dx.doi.org/10.1016/j.paid.2017.03.036

Villardón, L. (2009). El pensamiento suicida del adolescente. España: Universidad de Deusto.

Wahlbeck, K. (2015). Public mental health: the time is ripe for translation of evidence into practice. World Psychiatry, 14(1), 36-42. Doi:10.1002/wps.20178

Wahlstrom, K. \& Owens, J. (2017). School start time effects on adolescent learning and academic performance, emotional health and behaviour. Curr Opin Psychiatry, 30(6):485-490. Doi: 10.1097/YCO.0000000000000368

Wehrwein, E. \& Joyner, M. (2013). Regulation of blood pressure by the arterial baroreflex and autonomic nervous system. Handbook of Clinical Neurology. Recuperado de https://www.sciencedirect.com/science/article/pii/B9780444534910000080?via\%3Dih ub

Wu, J., Wu, Y., Feng, S., Meng, H., \& Chen, H. (2012). Mediating effects on depression regarding the relationship between negative life events and suicide ideation among college students. Zhonghua Liu Xing Bing Xue Za Zhi, (33), 1111-1114. Doi:10.3760/cma.j.issn.0254-6450.2012.11.002

Zanon, C., Roat, M. Cerentini, J. \& Hutz, C. (2013). Desenvolvimento e validação de uma escala de afetos positivos e negativos. Bragança Paulista. 18(2), 193-202. Recuperado de http://www.scielo.br/pdf/pusf/v18n2/v18n2a03.pdf

Zhang, J., Liu, Y., \& Sun L. (2017). Psychological strain and suicidal ideation: A comparison between Chinese and US college students. Psychiatry Res, (255), 256-262. Doi:10.1016/j.psychres.2017.05.046

Zhu, B., Chang, M., Mary, G. \& Kapella, C. (2019). Adaptation of the Pittsburgh Sleep Quality Index in Chinese adults with type 2 diabetes. Journal of the Chinese Medical Association, 81(3), 242-247. https://doi.org/10.1016/j.jcma.2017.06.021 